\title{
Contínuos Generalizados: de Voigt à Modelagem de Materiais Parcialmente Frágeis
}

\author{
Generalized Continuum: from Voigt to the \\ Modeling of Quasi-Brittle Materials
}

\author{
Jamile Salim Fuina ${ }^{1}$; Roque Luiz da Silva Pitangueira²; Samuel Silva Penna ${ }^{3}$
}

Resumo

Este artigo discute a utilização das teorias de contínuos generalizados para incorporar os efeitos da microestrutura na análise não linear, via Método dos Elementos Finitos, de materiais parcialmente frágeis e, assim, sanar problemas de dependência de malha. Inicia-se com uma descrição do problema denominado localização de deformações numericamente induzida, frequentemente encontrado em análises numéricas fisicamente não lineares via Método dos Elementos Finitos. A seguir, apresentase um breve histórico sobre os modelos baseados na Mecânica do Contínuo Generalizado, desde o trabalho inicial de Voigt (1887) até estudos mais recentes. Analisando esses modelos, observa-se que o contínuo de Cosserat e o contínuo com microexpansão são casos particulares de uma formulação geral que descreve o contínuo micromórfico. Após relatar tentativas de incorporação dos efeitos da microestrutura do material em modelos baseados na Mecânica do Contínuo Clássico, o artigo mostra a recente tendência de fazê-la segundo as premissas da Mecânica do Contínuo Generalizado. Por fim, apresentam-se resultados numéricos que permitem caracterizar esta tendência como um promissor caminho para solucionar o problema.

Palavras-chave: Contínuos generalizados. Meios parcialmente frágeis. Localização de deformações numericamente induzida. Método dos elementos finitos.

\begin{abstract}
This article discusses the use of the generalized continuum theories to incorporate the effects of the microstructure in the nonlinear finite element analysis of quasi-brittle materials and, thus, to solve mesh dependency problems. A description of the problem called numerically induced strain localization, often found in Finite Element Method material non-linear analysis, is presented. A brief historic about the Generalized Continuum Mechanics based models is presented, since the initial work of Voigt (1887) until the more recent studies. By analyzing these models, it is observed that the Cosserat and microstretch approaches are particular cases of a general formulation that describes the micromorphic continuum. After reporting attempts to incorporate the material microstructure in Classical Continuum Mechanics based models, the article shows the recent tendency of doing it according to assumptions of the Generalized Continuum Mechanics. Finally, it presents numerical results which enable to characterize this tendency as a promising way to solve the problem.

Key-words: Generalized continuums. Quasi-brittle media. Numerically induced strain localization. Finite element method.
\end{abstract}

1 Docente da Faculdade de Engenharia e Arquitetura da Universidade FUMEC. E-mail: jamile@fumec.br

2 Docente do Departamento de Engenharia de Estruturas da Universidade Federal de Minas Gerais. E-mail: roque@dees.ufmg.br

3 Doutorando do Programa de Pós-Graduação em Engenharia de Estruturas da Universidade Federal de Minas Gerais. E-mail: spenna@dees.ufmg.br 


\section{Introdução}

Materiais granulares e heterogêneos, normalmente formados a partir da combinação de diferentes constituintes, têm sido classificados como materiais parcialmente frágeis, devido à natureza de seu fraturamento. Dentre os materiais que fazem parte deste grupo, citam-se: argamassa, concreto simples, concreto armado, concreto com fibras (naturais ou artificiais) e outros geomateriais.

A complexidade do comportamento desses materiais é essencialmente causada pela composição de seus constituintes e por suas propriedades na microescala. A maneira com que a microestrutura do material afeta seu comportamento está, na maioria das vezes, relacionada à não uniformidade no tamanho, forma e rugosidade de suas partículas, entre outras características.

Em estruturas de materiais parcialmente frágeis, é comum o surgimento de problemas de fissuração, esmagamento, rigidez residual em regiões danificadas, perda de aderência, entre outros. $\mathrm{Na}$ análise numérica computacional do comportamento dessas estruturas, geralmente os materiais são considerados como inicialmente homogêneos, elásticos e isotrópicos, admitindo-se que, com a aplicação de cargas e consequentes deformações, os materiais deixam de ser elásticos e isotrópicos e tornam-se heterogêneos, pela degradação das regiões mais solicitadas. Neste tipo de análise, durante o processo de carregamento da estrutura, alguns pontos do domínio apresentam características mecânicas distintas dos demais, observando-se que esta combinação de materiais com características muito diversas - algumas regiões danificadas junto a outras com as características do material homogêneo inicial - causa efeitos não lineares pronunciados na resposta da estrutura.

A análise assim processada é incapaz de representar o complexo comportamento observado experimentalmente. Evidências experimentais demonstram que meios parcialmente frágeis, frequentemente, apresentam amolecimento ou "softening". Conforme Shah, Swartz e Ouyang (1995), a detecção e quantificação do "softening" vêm acompanhadas de localização de deformações. Este fenômeno se manifesta em uma zona de tamanho finito, dependente da microestrutura do meio, na qual as deformações crescem, enquanto o restante do volume estrutural experimenta descarregamento. Assim, a localização de deformações, enquanto fenômeno físico real, explica a obtenção de diferentes respostas tensão versus deformação em um mesmo experimento, dependendo da posição onde as deformações são medidas.

A aproximação clássica utilizada para representar o comportamento acima descrito consiste, simplesmente, em converter a curva carga-deslocamento, representativa da estrutura, numa curva tensão-deformação, representativa do ponto material. Numa análise numérica via Método dos Elementos Finitos (MEF), constata-se que este procedimento torna o problema de valor de contorno mal formulado, resultando em soluções dependentes do refinamento da malha (DE BORST, 1993). Além disso, para um caso limite de discretização, as deformações tendem a se localizar numa região de volume infinitesimal e a energia dissipada é incorretamente considerada nula (LI; TANG, 2005).

Este artigo discute a utilização das teorias de contínuos generalizados para incorporar os efeitos da microestrutura do material na resposta macroscópica de meios parcialmente frágeis e, assim, sanar os aludidos problemas de refinamento de malha.

\section{Localização de Deformações Numericamente Induzida}

Conforme dito acima, o uso da aproximação clássica na análise, via MEF, de meios parcialmente frágeis, tem apresentado diferentes curvas tensão versus deformação, dependendo da discretização utilizada. Esta dependência da malha também se explica pela ocorrência de localização de deformações. Entretanto, esta localização, 
doravante denominada localização de deformações numericamente induzida (LDNI), não tem nenhuma relação com a localização de deformações enquanto fenômeno físico real.

Para compreender a LDNI, toma-se, como exemplo, a análise não linear do ensaio de tração pura (Figura 1).

Como pode ser visto na Figura 1, mesmo considerando todos os elementos das malhas com as mesmas propriedades mecânicas, os resultados, representados por curvas carga $(\mathrm{F})$ versus deslocamentos $(\mathrm{u})$, são diferentes para cada discretização adotada. Ressalvando-se possíveis erros de discretização, essas diferenças podem estar associadas a alguma inconsistência no modelo constitutivo, fazendo com que as deformações se localizem em uma determinada região da malha, quando, para este caso, elas deveriam ser uniformes. Por exemplo: com refinamento da malha, o modelo constitutivo pode levar a uma superestimativa da rigidez em um determinado elemento finito, fazendo com que as deformações nele se localizem. Desta forma, quanto maior o número de elementos finitos, maior o "erro" introduzido. No caso ilustrado na Figura 1, se as deformações se localizam nas regiões destacadas, o comportamento dessas regiões é descrito pelo ramo descendente da curva tensão $(\sigma)$ versus deformação $(\varepsilon)$, enquanto as demais regiões da malha descarregam. Isto explica a obtenção de diferentes respostas para cada malha de elementos finitos utilizada, já que, em cada uma delas, a razão entre as dimensões das regiões carregadas e descarregadas é distinta. Esta falta de objetividade da malha tem sido atribuída (DE BORST, 1991, 1993; LAGES, 1997; PEERLINGS et al., 1998; LI; TANG, 2005; FUINA, 2009) à utilização do contínuo clássico e/ou de modelos constitutivos locais.
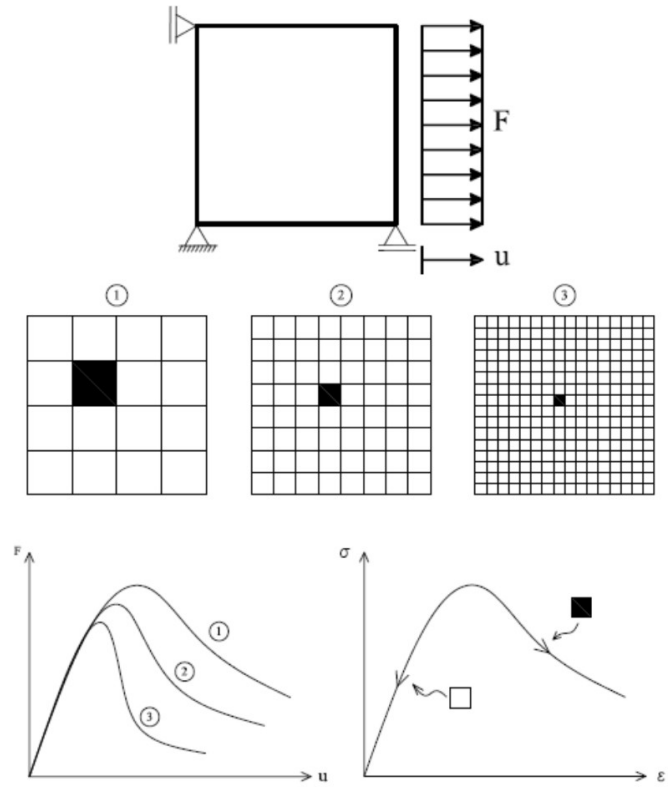

Figura 1. Localização numericamente induzida

Para simular de maneira correta a localização de deformações enquanto fenômeno físico real, caso esta ocorra pela existência de alguma região menos resistente do material, e impedir a ocorrência de localização de deformações numericamente induzida, é necessário introduzir algum mecanismo de regularização na teoria do contínuo clássico. Dentre os modelos com mecanismos de regularização citam-se os modelos constitutivos não locais (BAZANT; PIJAUDIER-CABOT, 1988) e os modelos que adicionam gradientes às variáveis de estado (AIFANTIS, 1984). Uma vez que no contínuo clássico as tensões e deformações são definidas localmente, seria preciso considerar uma região finita de envolvimento para o ponto do contínuo, definida por meio de uma dimensão característica. Para tanto, as teorias do contínuo generalizado, que possuem comprimentos característicos intrínsecos à sua concepção, podem ser utilizadas.

\section{Contínuos Generalizados: de Voigt (1887) à Fuina (2009)}

Os primeiros estudos visando incorporar efeitos da microestrutura do material na elasticidade 
referem-se ao trabalho de Voigt (1887). Neles, sugeria-se que a interação entre as partes materiais poderia se dar por meio de vetores de tensãomomento, além do clássico vetor de tensão.

Em 1909, os irmãos Eugène e François Cosserat apresentaram uma teoria da elasticidade assimétrica não linear (COSSERAT; COSSERAT, 1909). Porém, os conceitos discutidos não receberam a devida atenção, dada a complexidade da exposição.

Após um silêncio de aproximadamente meio século, os conceitos do "contínuo de Cosserat" foram retomados no notável trabalho do professor Günther (1958), que marcou o início de novas interpretações para os efeitos da microestrutura nos anos de 1960. No trabalho de Truesdell e Toupin (1960) foi apresentada uma teoria incompleta, revisada por Mindlin e Tiersten (1962), denominada, posteriormente, teoria do contínuo das tensões-momento (couple-stress theory). Nesta teoria, as microrrotações foram consideradas iguais às macrorrotações. Uma teoria linear mais geral é abordada no trabalho de Mindlin (1964), existindo um desacoplamento entre macrodeformações e microdeformações e dando origem à teoria do gradiente de deformação, na qual todos os componentes do primeiro gradiente de deformação são introduzidos na função de energia de deformação. Green e Rivlin (1964) estabeleceram as bases de uma teoria mais abrangente incluindo todos os gradientes de deformação de ordem superior na função de energia de deformação e a denominou teoria multipolar. Mindlin (1965) desenvolveu uma teoria em que somente o primeiro e o segundo gradientes de deformação são considerados, chamada teoria do segundo gradiente de deformação, como um caso especial da teoria multipolar.

No trabalho de Eringen (1966), apresentouse a teoria micropolar linear, caracterizada pela consideração de microrrotações independentes das macrorrotações. Uma coleção de trabalhos relacionados ao assunto foram apresentados no histórico simpósio IUTAM - International Union of Theoretical and Applied Mechanics (KRÖNER,
1968) sobre a Mecânica do Contínuo Generalizado, em 1967. Neste simpósio, Eringen (1968a) apresentou a teoria do contínuo micromórfico.

A primeira aplicação computacional não linear do contínuo micropolar em mecânica dos sólidos ocorreu nos trabalhos desenvolvidos por Mühlhaus (1989) e de Borst (1991), que analisaram os potenciais da teoria constitutiva micropolar elastoplástica. A partir daí, começaram a surgir vários trabalhos utilizando a teoria micropolar não linear para tratamento dos fenômenos de localização de deformações e/ou efeitos de tamanho (LAGES, 1997; ADHIKARY; DYSKIN, 1998; JOG, 2004; LI; TANG, 2005; LIU; HU, 2005; VOYIADJIS; ALSALEH; ALSHIBLI, 2005; HU; LIU; LU, 2005; FUINA, 2009).

Atualmente, a teoria micropolar é usada em descrições macroscópicas de materiais com microestrutura rígida, incluindo cristais líquidos, meios granulares e materiais porosos (ERINGEN, 1997; WALSH; TORDESILLAS, 2004; LIU; SCARPAS; KASBERGEN, 2007). Além disso, formulações do contínuo micropolar têm sido, nas últimas décadas, intensamente estudadas dentro do contexto de localização de deformações, constatando-se que, na modelagem numérica do comportamento de materiais que apresentam o fenômeno de amolecimento, a introdução da microrrotação como grau de liberdade tem provocado um efeito de regularização do problema de dependência da malha de elementos finitos durante o processo de amolecimento (MÜHLHAUS; VARDOULAKIS, 1987; DE BORST, 1991). Entretanto, ressalta-se que a microrrotação somente é ativada sob carregamento cisalhante e o referido efeito de regularização não é esperado na solução de problemas de estado de tração dominante, uma vez que, neste caso, a microrrotação não é ativada (KIRCHNER; STEINMANN, 2007).

Alguns autores (DE BORST; MÜHLHAUS, 1992; SLUYS, 1992) destacam que a utilização do contínuo de Cosserat não é adequada quando predomina o modo de abertura (modo I na 
linguagem de Mecânica da Fratura) nas localizações de deformações e sugerem o contínuo com microexpansão como solução para este problema.

O contínuo com microexpansão pertence à classe de contínuos micromórficos. Sua microestrutura não é tratada como rígida, como no caso do contínuo de Cosserat, mas pode apresentar, além de microrrotações, expansões (ou contrações) isotrópicas. Sua utilização é recomendada porque, além de aproveitar os benefícios trazidos pelo contínuo de Cosserat, o qual tem apresentado resultados satisfatórios para a modelagem de localização de deformações com predominância do modo de cisalhamento (DE BORST, 1991, 1993; DE BORST; SLUYS, 1991; RISTINMAA; VECCHI, 1996; LAGES, 1997), o contínuo com microexpansão permite considerar também a ocorrência de descontinuidades nos campos das deformações normais.

O desenvolvimento de novos modelos constitutivos utilizando-se estes contínuos é de suma importância para tentar resolver problemas de localização de deformações numericamente induzida. Esta tentativa foi feita por Fuina (2009), que desenvolveu dois modelos constitutivos, um que utiliza a descrição cinemática do contínuo de Cosserat e outro que utiliza a descrição cinemática do contínuo com microexpansão, ambos com descrição estática baseada nos modelos de microplanos (BAZANT; GAMBAROVA, 1984; CAROL; PRAT; BAZANT, 1992; OZBOLT; LI; KOZAR, 2001; LEUKART; RAMM, 2006).

\section{Formulação Geral e Casos Particulares}

Diferentemente da mecânica do contínuo clássico, onde o movimento (macromovimento) de uma partícula material é totalmente descrito por translações na macroescala, as partículas materiais micromórficas sofrem um micromovimento adicional, correspondente à rotação e deformação da partícula material na microescala (os microcontínuos). De acordo com Eringen (1968a) e como ilustrado na Figura 2, este micromovimento pode ser descrito por um tensor $\left(\chi_{i j}\right)$ que introduz nove graus de liberdade adicionais: três microrrotações e seis microdeformações, que provocam mudança de volume e de forma dos microcontínuos.

A Figura 2 também mostra que a teoria micromórfica possui dois casos especiais que são obtidos ao se aplicar restrições ao tensor $\chi_{i j}$. O primeiro é o contínuo com microexpansão (ERINGEN, 1990), que possui quatro graus de liberdade adicionais em relação ao clássico, três microrrotações $\left(\phi_{i}\right)$ e uma microexpansão $(\varphi)$, isto é, seus microcontínuos podem sofrer expansão ou contração isotrópica, mas não podem sofrer mudança de forma. O segundo é o contínuo de Cosserat - ou contínuo micropolar (COSSERAT; COSSERAT, 1909; TOUPIN, 1962; ERINGEN, 1968b), que é um caso especial de contínuo com microexpansão, e consequentemente, de contínuo micromórfico, apresentando somente mais três microrrotações $\left(\phi_{i}\right)$ em relação ao clássico. Para o contínuo micropolar, os componentes $\chi_{i j}$ são considerados rígidos e até a mudança de volume dos microcontínuos é impedida.

Como mencionado anteriormente, a teoria do contínuo micromórfico, bem como seus casos especiais, se destaca por inserir novos graus de liberdade ao contínuo clássico, enquanto as demais se destacam por associar a função de energia aos gradientes de deformação. Contudo, existem relações entre estes dois conjuntos de teorias do contínuo generalizado, conforme mostra a Figura 2. Nesta figura, pode-se perceber que a teoria micropolar se reduz à teoria das tensões-momento quando se admite a igualdade entre a micro e a macrorrotação, $\phi_{k}=\epsilon_{i j k} u_{j, i} / 2$. Esta, por sua vez, é um caso especial de teoria do gradiente de deformação, pois somente o gradiente do vetor rotação entra na função de energia de deformação, isto é, apenas alguns componentes do primeiro gradiente de deformação. A teoria micromórfica também se reduz à teoria do gradiente de deformação se $\chi_{i j}$ forem definidos iguais ao gradiente de deslocamento $u_{i, j}$, neste caso, ocorre a fusão do meio micro com o meio macro. 


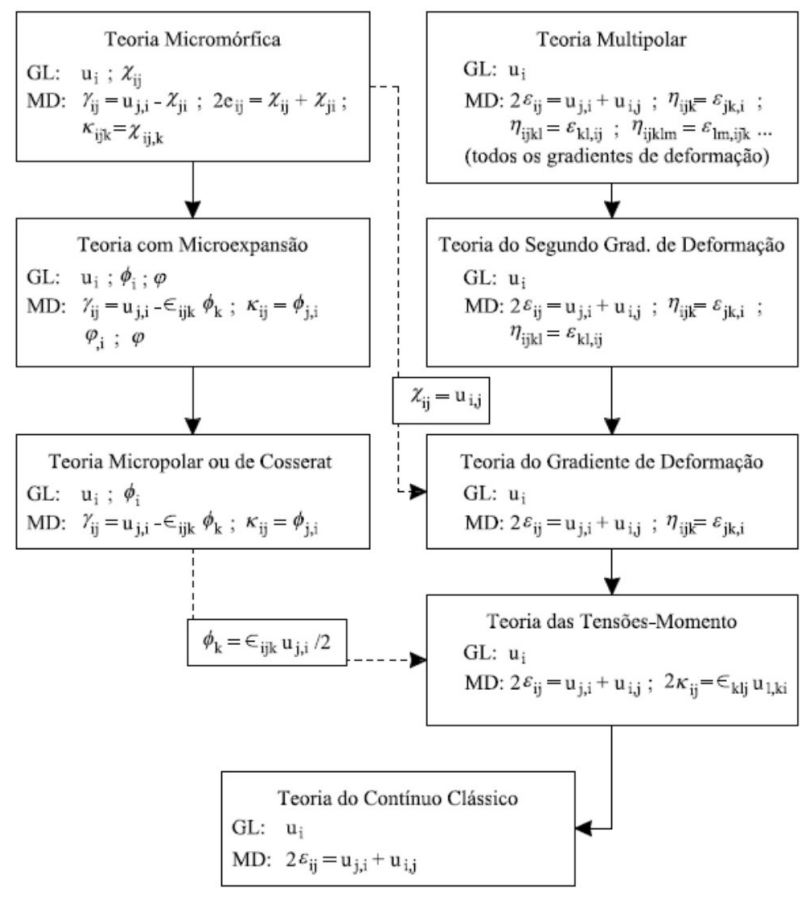

Figura 2. Relações entre as teorias do contínuo generalizado

\section{SIMBOLOGIA:}

GL - grau de liberdade;

MD - medida de deformação;

$u_{i}$ - vetor de deslocamentos;

$\chi_{i j}$ - tensor que contém os graus de liberdade dos microcontínuos;

$\gamma_{i j}$ - tensor associado a variações das dimensões e distorções;

$\kappa_{i j k}$ - tensor gradiente de microdeformações;

$e_{i j}$ - tensor relacionado às medidas de deformação dos microcontínuos;

$\phi_{i}-$ vetor de microrrotação;

$\varphi$ - expansão (ou contração) volumétrica;

$\epsilon_{i j k}$ - tensor alternante;

$\kappa_{i j}$ - tensor relacionado às curvaturas e às torções da microestrutura;

$\varepsilon_{i j}$ - clássico tensor de deformações;

$\eta_{i j k}$ - tensor gradiente de deformações;

\section{Contínuos Micromórficos}

$\mathrm{Na}$ teoria do contínuo clássico, o meio é considerado uma distribuição contínua de partículas, cada uma representada geometricamente por um ponto material $\mathbf{X}$ de coordenadas cartesianas, com referência a um sistema fixo de eixos ortogonais $X_{i}(i=1,2,3)$. Além disso, tais partículas são caracterizadas cinematicamente por um vetor de deslocamentos. Já no contínuo micromórfico, cada ponto é visto como uma partícula de dimensão reduzida, a qual constitui, por si própria, um pequeno contínuo em torno do ponto $\mathbf{X}$. Por conseguinte, a caracterização cinemática dessa partícula requer um refinamento com relação àquela do contínuo clássico. A diferença entre os contínuos clássicos e os contínuos generalizados se estende à descrição constitutiva do material, que considera a presença de um comprimento intrínseco representando a dimensão da partícula. Caso o comprimento intrínseco seja desprezível, o contínuo generalizado degenerar-se-á para um contínuo clássico. No caso contrário, se for significativo, não será possível descrever a cinemática das partículas segundo um contínuo convencional.

Um corpo micromórfico é composto por uma coleção de microcontínuos chamados microrregiões (Figura 3).

Em sua configuração de referência, a macrorregião considerada apresenta volume $\Delta V$ e superfície $\Delta S$, supondo-se que seja constituída por $\mathrm{N}$ microrregiões $\Delta V^{(\alpha)}+\Delta S^{(\alpha)}(\alpha=1,2, \ldots, \mathrm{N})$. Nesta configuração, $\mathbf{X}$ corresponde à posição de referência do centro de massa $\mathrm{P}$ da macrorregião e $\mathbf{X}^{(\alpha)}$ à posição de referência do centro de massa $Q^{(\alpha)}$ da microrregião e $\overline{\mathbf{X}}^{(\alpha)}$ corresponde à posição de referência de $Q^{(\alpha)}$ com relação a P. Sob a influência de cargas externas, o corpo se deforma e a macrorregião ocupa um novo volume $\Delta \mathrm{v}$ de superfície $\Delta \mathrm{s}$. 


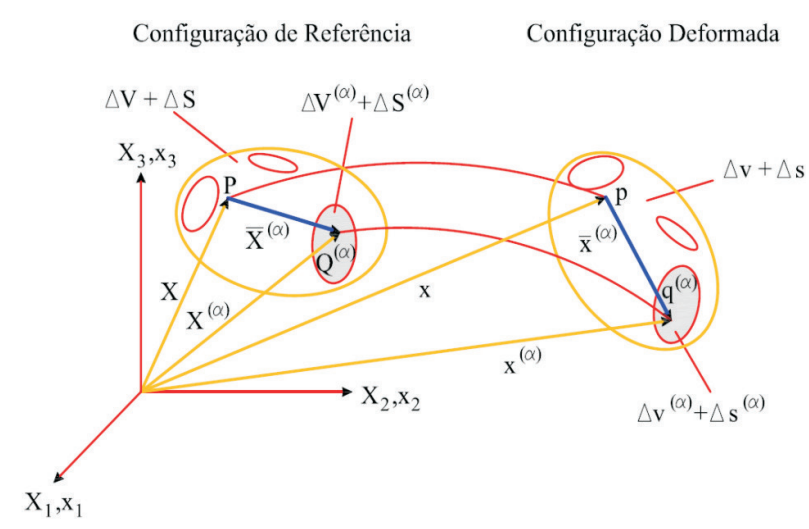

Figura 3. Cinemática do contínuo micromórfico

As medidas de deformação são definidas estudando-se um segmento diferencial em uma microrregião $\alpha$ na configuração deformada, onde esta sofre um microdeslocamento generalizado. Algumas teorias aplicam restrições a este microdeslocamento, entre elas, a teoria do contínuo de Cosserat. Neste caso, a restrição é feita de forma a descrever um movimento de corpo rígido correspondente à uma rotação em torno do centro de massa do macrovolume na configuração deformada (ponto p na Figura 4), onde $\overline{\mathbf{u}}^{\text {pepresenta } \mathrm{o}}$ microdeslocamento e $\phi$ as microrrotações.

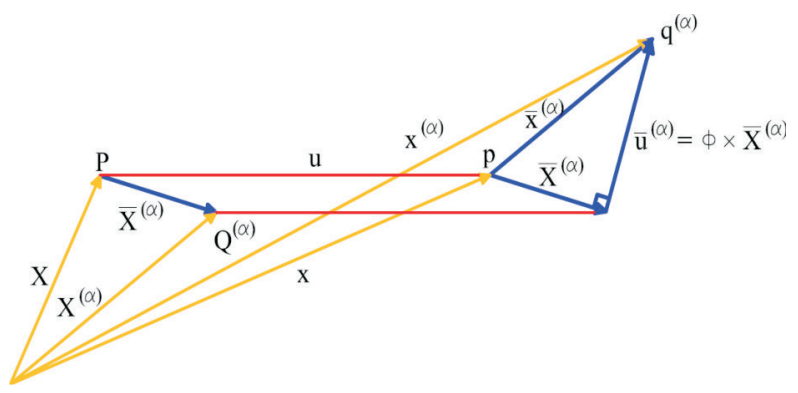

Figura 4. Cinemática do contínuo de Cosserat

Assim, as informações tensoriais necessárias para definir as deformações do contínuo de Cosserat são

$$
\begin{aligned}
\gamma_{i j} & =u_{j, i}-\epsilon_{i j k} \phi_{k} \\
\kappa_{i j} & =\phi_{j, i}
\end{aligned}
$$

onde $\epsilon_{i j k}$ representa o tensor alternante, $u_{i}$ os macrodeslocamentos e $\phi_{k}$ as microrrotações. O tensor $\gamma_{i j}$ está associado a variações das dimensões e distorções, enquanto o tensor $\kappa_{i j}$ está relacionado às curvaturas e às torções da microestrutura.

Uma outra teoria que aplica restrições ao microdeslocamento do contínuo micromórfico é a teoria do contínuo com microexpansão. Neste caso, a restrição é feita de forma a descrever uma expansão (ou contração) isotrópica combinada com um movimento de corpo rígido correspondente à uma rotação em torno do centro de massa do macrovolume na configuração deformada (ponto $\mathbf{p}$ na Figura 5).

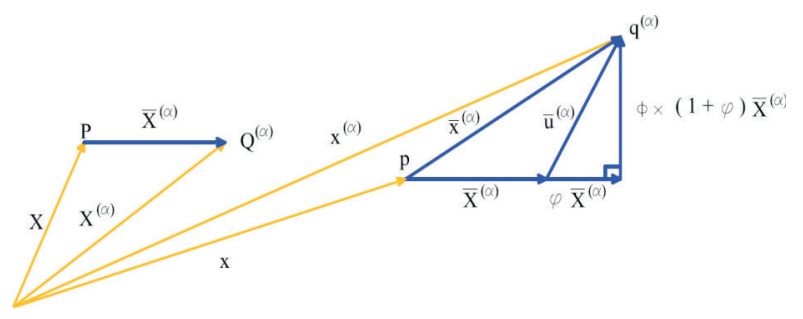

Figura 5. Cinemática do contínuo com microexpansão

Assim, as informações tensoriais necessárias para definir as deformações do contínuo com microexpansão são

$$
\begin{aligned}
& \gamma_{i j}=u_{j, i}-\epsilon_{i j k} \phi_{k} \\
& \kappa_{i j}=\phi_{j, i} \\
& \varphi \\
& \varphi_{, i}
\end{aligned}
$$

onde os dois primeiros tensores já são conhecidos da teoria do contínuo micropolar e a microexpansão volumétrica $\varphi$ e seu gradiente $\varphi_{, i}$ são consideradas medidas de deformações independentes.

\section{Modelagem de Meios Parcialmente Frágeis e Teorias do Contínuo Generalizado}

O estudo de meios parcialmente frágeis, considerando-os como contínuos e homogêneos, 
tem sido feito pela caracterização de uma dimensão estrutural, suficientemente grande para o tratamento como contínuo e suficientemente pequena para a ocorrência de "softening" estável - redução da capacidade resistente acompanhada de aumento de deformações —, que é função da natureza heterogênea do material. Tal dimensão é muitas vezes referida como comprimento característico do material (PITANGUEIRA, 1998).

A introdução de uma dimensão característica na análise via Método dos Elementos Finitos é devida a Hillerborg, Modéer e Peterson (1976), que utilizaram o modelo de fissuras discretas. Baseando-se nesta proposição, Bazant e Oh (1983) formularam o modelo de banda de fissuração com inclusão de parâmetros de Mecânica da Fratura e comprimento característico.

A associação das características geométricas da discretização com o comprimento característico do material (BAZANT; OH, 1983; BAZANT, 1986) reflete a preocupação em representar a natureza heterogênea do material, quando o mesmo é tratado como contínuo e homogêneo. Entretanto, esta prática não é recomendada, pois o comprimento característico é uma propriedade do material e não uma grandeza associada ao tamanho dos elementos finitos.

Alguns autores optam pela utilização de um comprimento característico em um enfoque não local (BAZANT; PIJAUDIER-CABOT, 1988). A hipótese central deste enfoque é que a tensão em um ponto não é uma função da deformação neste mesmo ponto, mas uma função das deformações em um determinado volume centrado no referido ponto, cujas dimensões dependem do comprimento característico.

Mais recentemente, o enfoque não local tem sido usado, juntamente com o modelo constitutivo de microplanos. Em lugar de especificar as propriedades constitutivas segundo direções ortogonais, vários pesquisadores (BAZANT; GAMBAROVA, 1984; CAROL; PRAT; BAZANT,
1992; OZBOLT; LI; KOZAR, 2001) têm proposto especificar tais propriedades por meio de relações tensão-deformação admitidas válidas sobre planos de orientação arbitrária no material, os microplanos.

As iniciativas acima descritas têm uma característica em comum: tentam incorporar o comprimento característico do material em modelos baseados na Mecânica do Contínuo Clássico. Isto é feito por meio de alterações na descrição estática do meio, seu modelo constitutivo, sem qualquer modificação em sua descrição cinemática, gerando modelos energeticamente inconsistentes.

Visando remediar a aludida inconsistência, vários estudos têm proposto conjugar descrições estáticas consagradas com teorias do contínuo micromórfico, que permitem considerar, nas relações constitutivas macroscópicas, o comportamento da microestrutura do material. Como exemplos destes estudos citam-se os modelos elastoplásticos do contínuo micropolar (MÜHLHAUS; VARDOULAKIS, 1987; MÜHLHAUS, 1989; DE BORST, 1991; LAGES, 1997) e os modelos de microplanos para contínuos generalizados (FUINA, 2009). Nestes modelos, a inclusão do comprimento característico surge naturalmente com a descrição cinemática do contínuo.

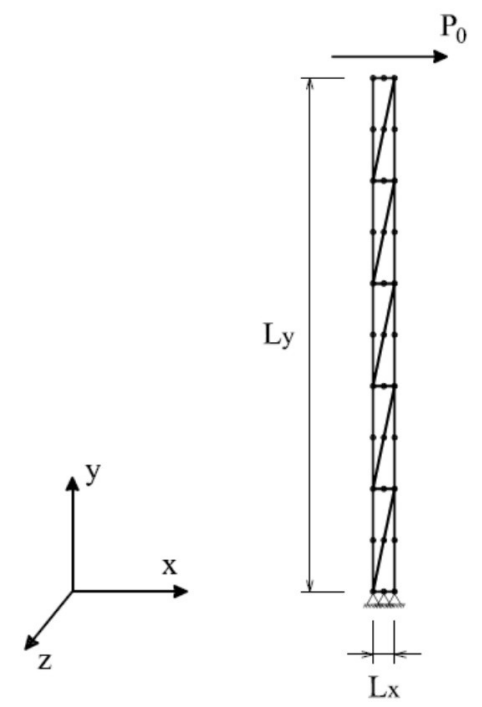

Figura 6. Camada infinita sob cisalhamento 
Para exemplificar o promissor caminho oferecido pelos modelos baseados na Mecânica do Contínuo Generalizado, apresenta-se aqui a modelagem de uma camada infinita sob cisalhamento, adotandose a descrição estática elastoplástica conjugada com a teoria do contínuo micropolar, cujos detalhes podem ser encontrados nos trabalhos de De Borst (1991,1993), Lages (1997), Li e Tang (2005) e Fuina (2009). Uma camada (Figura 6) no plano $x y$ de altura $\mathrm{L}_{\mathrm{y}}=100 \mathrm{~mm}$ e $\operatorname{largura} \mathrm{L}_{\mathrm{x}}=$ 2,5 $\mathrm{mm}$, infinitamente longa na direção do eixo $z$, é submetida a um carregamento distribuído de cisalhamento em sua borda superior, a camada é modelada como um problema em estado plano de deformação, utilizando-se elementos finitos triangulares de 6 nós. Os parâmetros do modelo são os mesmos utilizados por De Borst (1991, 1993), a saber: módulo de elasticidade $(\mathrm{E})=10000 \mathrm{MPa}$; coeficiente de Poisson $(v)=0,25$; módulo plástico $(\mathrm{h})=-500 \mathrm{MPa}$; tensão de escoamento inicial $(\bar{\sigma})=100 \mathrm{MPa}$; comprimento característico à flexão $\left(L_{f}\right)=12 \mathrm{~mm}$ e módulo de cisalhamento rotacional $(\alpha)=2000 \mathrm{MPa}$.

Os resultados mostrados nas Figuras 7 e 8 foram obtidos utilizando-se a implementação computacional feita no sistema INSANE (Interactive Structural Analysis Environment), segundo Fuina (2009), para malhas com 10, 20, 100 e 200 elementos finitos.

Na Figura 7 são mostradas as curvas fator de carga versus deslocamento horizontal da borda superior da camada. Tais curvas foram obtidas utilizandose o método de controle direto de deslocamento (BATOZ; DHAT, 1979), incrementando-se de $0,1 \mathrm{~mm}$ o deslocamento horizontal do nó central da borda superior da camada, adotando-se uma tolerância para o erro de convergência em força de $10^{-4}$. Em todos os casos, as trajetórias de equilíbrio foram interrompidas por divergência no processo iterativo.
Na Figura 8, a deformada e a distribuição de deformações cisalhantes $\gamma_{y x}$ correspondem ao menor nível do fator de carga do ramo descendente comum às quatro discretizações adotadas.

Na Figura 7, observa-se que, com o refinamento da malha, as curvas fator de carga versus deslocamento convergem para uma única solução, indicando a objetividade do modelo quanto ao tamanho dos elementos finitos. Percebe-se também que a curva obtida para a discretização de 10 elementos finitos (malha mais grosseira) é um pouco diferente das demais, concluindo-se que esta malha é inadequada para representar o problema. Para as demais discretizações observa-se que, independente da malha de elementos finitos, a extensão da zona de localização, apesar de apresentar tamanhos diferentes, abrange uma determinada região (Figura 8), cuja dimensão está associada ao tamanho característico do material.

A diferença entre os resultados obtidos com estas discretizações é refletida pelo tamanho da zona de localização, que pode ser identificada pela região de maior nível de deformação, conforme detalhe da Figura 8. A dimensão dessa região tende para um valor único, que representa a configuração da zona de localização.

Este fato mostra que o modelo, ao incorporar, de forma estática e cinematicamente consistente, o comprimento característico do material, é capaz de representar de maneira correta a localização de deformação enquanto fenômeno físico real: aquele que, associado à heterogeneidade do meio, define a zona de processamento da degradação, experimentalmente encontrada em meios parcialmente frágeis.

Observa-se também, na Figura 8, que o nível de deformação específica é elevado (aproximadamente 65 partes por mil), sugerindo, assim, uma análise geometricamente não linear. 


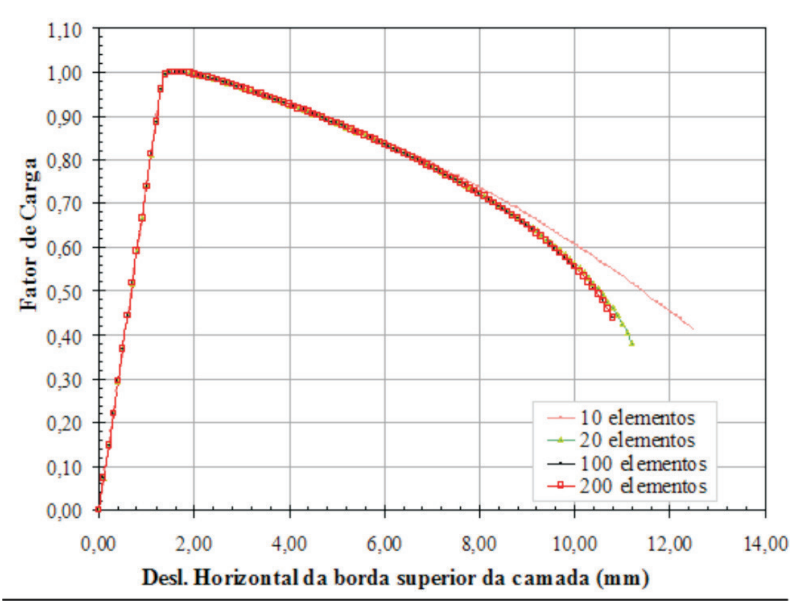

Figura 7. Trajetórias de equilíbrio correspondentes ao deslocamento horizontal da borda superior da camada infinita para malhas de 10, 20, 100 e 200 elementos

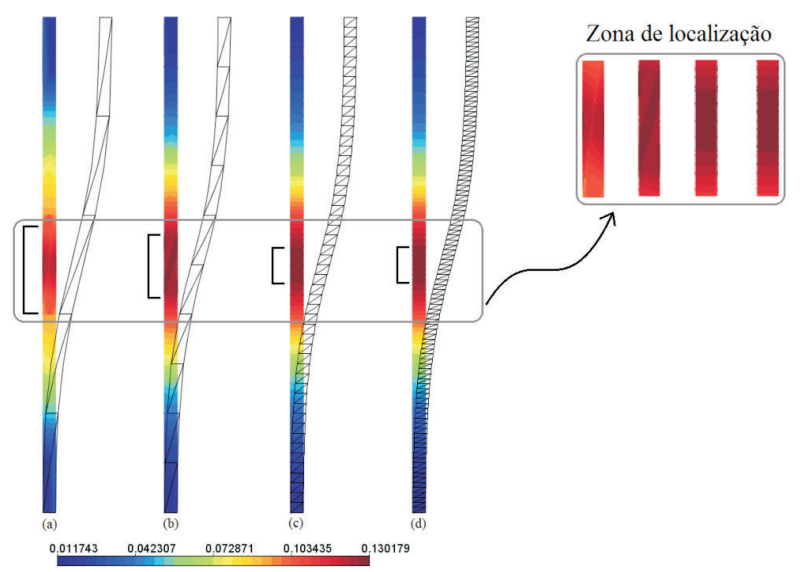

Figura 8. Deformada e distribuição de deformações cisalhantes $\gamma_{v x}$ para (a) 10 elementos, (b) 20 elementos, (c) 100 elementos e (d) 200 elementos

\section{Considerações Finais}

Modelar o fenômeno de amolecimento por meio do contínuo clássico pode resultar numa escolha pouco representativa da realidade física. Numericamente, isto se manifesta na dependência patológica da malha de elementos finitos, ou seja, localizações que inevitavelmente acompanham os processos de falha nos materiais parcialmente frágeis tendem a ser determinadas inteiramente pelo espaçamento da malha de elementos finitos.

Inúmeras aproximações têm sido propostas para enriquecer a descrição do contínuo tal que a localização possa se desenvolver corretamente
(BAZANT; PIJAUDIER-CABOT, 1988; MÜHLHAUS; VARDOULAKIS, 1987; DE BORST, 1991,1993).

As estratégias baseadas no contínuo micropolar têm apresentado resultados satisfatórios para a modelagem de localização de deformações com predominância do modo de cisalhamento (ou modo II). Entretanto, alguns autores comentam a respeito de sua inaplicabilidade no caso de predominância do mecanismo de localização no modo de abertura (ou modo I), muito importante no caso de concreto e materiais geológicos (LAGES, 1997). Neste caso, o contínuo com microexpansão surge como uma alternativa, pois além de conter a teoria micropolar, também possui a microexpansão volumétrica como grau de liberdade adicional, tornando-se capaz de representar também o modo I de abertura de fissuras. Isso ocorre porque este contínuo possui comprimentos característicos à flexão e à tração.

A vantagem de se ter um sistema computacional no qual se possa optar entre o contínuo com microexpansão e o micropolar está na possibilidade de escolher o contínuo mais adequado para representar as deformações da microestrutura do material, quando conhecidas a priori. Outro fato que deve ser considerado é o desempenho numérico, pois em problemas bidimensionais, o contínuo com microexpansão possui 6 grandezas internas adicionais em relação ao contínuo micropolar (uma microexpansão, seus dois gradientes, uma microtensão e duas microforças), o que acarreta um custo computacional maior no cálculo das tensões e deformações e principalmente no armazenamento dessas grandezas. Logo, a utilização do contínuo micropolar deve ser preferida quando não houver necessidade do uso do contínuo com microexpansão.

\section{Agradecimentos}

Os autores agradecem o apoio financeiro em forma de fomento à pesquisa concedido pela FAPEMIG (Fundação de Amparo à Pesquisa do Estado de Minas Gerais) e pela CAPES 
(Coordenação de Aperfeiçoamento de Pessoal de Nível Superior).

\section{Referências}

ADHIKARY, D. P.; DYSKIN, A. V. A continuum model of layered rock masses with non-associative joint plasticity. International Journal for Numerical and Analytical Methods in Geomechanics, Chichester, v. 22, n. 4, p. 245-261, 1998.

AIFANTIS, E. C. On the microstructural origin of certain inelastic models. ASME Journal of Engineering Materials and Technology, New York, v. 106, n. 4, p. 326-330, 1984.

BATOZ, J. L.; DHAT, G. Incremental displacement algorithms for nonlinear problems. International Journal for Numerical Methods in Engineering, Chichester, v. 14, n. 8, p. 1262-1267, 1979.

BAZANT, Z. P. Mechanics of distributed cracking. Applied Mechanics Review, Easton, v. 39, n. 5, p. 675705, 1986.

BAZANT, Z. P.; GAMBAROVA, P. G. Crack shear in concrete: Crack band microplane model. Journal of Structural Engineering, New York, v. 110, n. 9, p. 20152035, 1984.

BAZANT, Z. P.; OH, B. H. Crack band theory for fracture of concrete. Matériaux et Constructions, Paris, v. 16, n. 93, p. 155-177, 1983.

BAZANT, Z. P.; PIJAUDIER-CABOT, G. Nonlocal continuum damage, localization instability and convergence. ASME Journal of Applied Mechanics, New York, v. 55, n. 2, p. 287-293, 1988.

CAROL, I.; PRAT, P. C.; BAZANT, Z. P. New explicit microplane model for concrete: theoretical aspects and numerical implementation. International Journal of Solids and Structures, New York, v. 29, n. 9, p. 11731191, 1992.

COSSERAT, E.; COSSERAT, F. Théorie des corps déformables. Paris: Hermann \& Fils, 1909.

DE BORST, R. A generalisation of $\mathrm{J}_{2}$-flow theory for polar continua. Computer Methods in Applied Mechanics and Engineering, Amsterdam, v. 103, n. 3, p. 347-362, 1993.

Simulation of strain localization: a reappraisal of the Cosserat continuum. Engineering Computations, Swansea, v. 8, n. 4, p. 317-332, 1991.

DE BORST, R.; MÜHLHAUS, H.-B. Gradientdependent plasticity: Formulation and algorithmic aspects. International Journal for Numerical Methods in Engineering, Chichester, v. 35, n. 3, p. 521-539, 1992.

DE BORST, R.; SLUYS, L. J. Localisation in a Cosserat continuum under static and dynamic loading conditions. Computer Methods in Applied Mechanics and Engineering, Amsterdam, v. 90, p. 805-827, 1991.

ERINGEN, A. C. Linear theory of micropolar elasticity. Journal of Mathematics and Mechanics, Bloomington, v. 15, p. 909-923, 1966.

Mechanics of micromorphic continua. In: KRÖNER, E. (Ed.). IUTAM Symposium on generalized continuum. Nova Iorque: Springer-Verlag, 1968a. p. 1835.

. Theory of micropolar elasticity. In: LEIBOWITZ, H. II - Mathematical fundamentals. Nova Iorque: Academic Press, 1968b. cap. 7, p. 621-729.

Theory of thermo-microstretch elastic solids. International Journal of Engineering Science, Elmsford, v. 28, n. 12, p. 1291-1301, 1990.

. A unified continuum theory of electrodynamics of liquid crystals. International Journal of Engineering Science, Elmsford, v. 35, n. 12-13, p. 1137-1157, 1997.

FUINA, J. S. Formulações de modelos constitutivos de microplanos para contínuos generalizados. 2009. Tese (Doutorado em Engenharia de Estruturas) Universidade Federal de Minas Gerais, Belo Horizonte.

GREEN, A. E.; RIVLIN, R. S. Multipolar continuum mechanics. Archive for Rational Mechanics and Analysis, New York, v. 17, n. 2, p. 113-147, 1964.

GÜNTHER, W. Zur statik und kinematik des Cosseratschen kontinuums. In: __. Abhandlungen der koniglichen gesellschaft $\overline{\text { der wissenchaften } z u}$ Göttingen. Göttingen: Dieterichschen Buchhandlung, 1958. v. 10. p. 195-213.

HILlERBORG, A.; MODÉER, M.; PETERSON, P. Analysis of crack formation and crack growth in concrete by means of fracture mechanics and finite elements. Cement and Concrete Research, Elmsford, v. 6, n. 6, p. 773-782, 1976.

HU, G.; LIU, X.; LU, T. J. A variational method for nonlinear micropolar composites. Mechanics of Materials, Amsterdam, v. 37, n. 4, p. 407-425, 2005.

JOG, C. S. Higher-order shell elements based on a cosserat model, and their use in the topology design of structures. Computer Methods in Applied Mechanics and Engineering, Amsterdam, v. 193, n. 23/26, p. 2191-2220, 2004.

KIRCHNER, N.; STEINMANN, P. Mechanics of extended continua: Modeling and simulation of elastic 
microstretch materials. Computational Mechanics, Berlin, v. 40, n. 4, p. 651-666, 2007.

KRÖNER, E. (Ed.). Proceedings of the International Union of Theoretical and Applied Mechanics Symposium on the Generalized Cosserat Continuum and the Continuum Theory of Dislocations with Applications. IUTAM - International Union of Theoretical and Applied Mechanics. Stuttgart: Springer-Verlag, 1968.

LAGES, E. N. Modelagem de localização de deformações com teorias de contínuo generalizado. 1997. Tese (Doutorado em Engenharia Civil) — Pontifícia Universidade Católica do Rio de Janeiro, Rio de Janeiro.

LEUKART, M.; RAMM, E. Identification and interpretation of microplane material laws. Journal of Engineering Mechanics, New York, v. 132, n. 3, p. 295305, 2006.

LI, X.; TANG, H. A consistent return mapping algorithm for pressure-dependent elastoplastic cosserat continua and modelling of strain localisation. Computers \& Structures, Elmsford, v. 83, n. 1, p. 1-10, 2005.

LIU, X.; HU, G. A continuum micromechanical theory of overall plasticity for particulate composites including particle size effect. International Journal of Plasticity, New York, v. 21, n. 4, p. 777-799, 2005.

LIU, X.; SCARPAS, A.; KASBERGEN, C. A micropolar formulation of the Desai hierarchical model for elastoplastic porous media. International Journal of Solids and Structures, New York, v. 44, n. 9, p. 2695 2714, 2007.

MINDLIN, R. D. Micro-structure in linear elasticity. Archive for Rational Mechanics and Analysis, New York, v. 16, n. 1, p. 51-78, 1964.

Second gradient of strain and surface-tension in linear elasticity. International Journal of Solids and Structures, New York, v. 1, n. 4, p. 417-438, 1965.

MINDLIN, R. D.; TIERSTEN, H. F. Effects of couplestresses in linear elasticity. Archive for Rational Mechanics and Analysis, New York, v. 11, n. 1, p. 415448, 1962.

MÜHLHAUS, H.-B. Application of cosserat theory in numerical solutions of limit load problems. IngenieurArchiv, New York, v. 59, n. 2, p. 124-137, 1989.

MÜHLHAUS, H.-B.; VARDOULAKIS, I. The thickness of shear bands in granular materials. Géotechnique, Ottawa, v. 37, n. 3, p. 271-283, 1987.

OZBOLT, J.; LI, Y.; KOZAR, I. Microplane model for concrete with relaxed kinematic constraint. International Journal of Solids and Structures, New York, v. 38, n. 16, p. 2683-2711, 2001.
PEERLINGS, R. H. J.; DE BORST, R.; BREKELMANS, W. A. M.; GEERS, M. G. D. Gradient-enhanced damage modelling of concrete fracture. Mechanics of CohesiveFrictional Materials, Chichester, v. 3, n. 4, p. 323-342, 1998.

PITANGUEIRA, R. L. S. Mecânica de estruturas de concreto com inclusão de efeitos de tamanho e heterogeneidade. 1998. Tese (Doutorado em Engenharia Civil) - Pontificia Universidade Católica do Rio de Janeiro, Rio de Janeiro.

RISTINMAA, M.; VECCHI, M. Use of couple-stress theory in elasto-plasticity. Computer Methods in Applied Mechanics and Engineering, Amsterdam, v. 136, n. 3/4, p. 205-224, 1996.

SHAH, S. P.; SWARTZ, S. E.; OUYANG, C. Fracture mechanics of concrete: applications of fracture mechanics to concrete rock, and other quasi-brittle materials. Nova Yorque: John Wiley \& Sons, 1995.

SLUYS, L. J. Wave propagation, localisation and dispersion in softening solids. 1992. Thesis (PhD in Engineering) - Delft University of Technology, Delft, 1992.

TOUPIN, R. A. Elastic materials with couple-stresses. Archive for Rational Mechanics and Analysis, New York, v. 11, n. 1, p. 385-414, 1962.

TRUESDELL, C.; TOUPIN, R. A. The classical field theories. In: FLÜGGE, S. (Ed.). Handbuch der physik. Berlin: Springer-Verlag, 1960. v. III-1.

VOIGT, Waldemar. Theoretische studien über die elasticitätsverhältnisse der krystalle. In: Abhandlungen der koniglichen gesellschaft der wissenchaften zu Göttingen. Göttingen: Dieterichschen Buchhandlung, 1887. v. 34. p. 3-100.

VOYIADJIS, G. Z.; ALSALEH, M. I.; ALSHIBLI, K. A. Evolving internal length scales in plastic strain localization for granular materials. International Journal of Plasticity, New York, v. 21, n. 10, p. 2000-2024, 2005.

WALSH, S. D. C.; TORDESILLAS, A. A thermomechanical approach to the development of micropolar constitutive models of granular media. Acta Mechanica, New York, v. 167, n. 3/4, p. 145-169, 2004.

Recebido em 14 Dezembro, 2009 - Received on December 14, 2009. Aceito em 23 junho, 2010-Accepted on June 23, 2010. 